\title{
Keck Segment Surface Artifacts: Impacts on Segment Phasing and Image Quality
}

\author{
Mitchell Troy, ${ }^{a}$ Gary Chanan, ${ }^{b}$ and Neal Brock ${ }^{c}$ \\ ${ }^{a}$ Jet Propulsion Laboratory, California Institute of Technology, Pasadena, CA 91109 \\ ${ }^{b}$ Department of Physics and Astronomy, University of California Irvine, Irvine, CA 92697 \\ ${ }^{c} 4 \mathrm{D}$ Technology, Tucson, AZ 85706
}

\begin{abstract}
We show that the segments of the Keck telescopes suffer from small but significant surface artifacts that limit segment phasing accuracy. Both the segment surface artifacts themselves and the limits they place on segment phasing have important implications for adaptive optics, high contrast imaging, and seeing limited observations. These surface artifacts are likely by-products of the segment polishing, most likely the Ion Beam Figuring (IBF) process that constitutes the final step of the segment surface figuring. The surface artifacts diffract a significant amount of light out past \pm 3.5 arcseconds and generate chromatic effects that limit segment phasing accuracy. We present on-sky measurements of the diffracted intensity. Segment surface roughness measurements are also presented, which show that surface roughness is not the source of the problem. These results have direct relevance to segment manufacturing for the Thirty Meter Telescope (TMT) and other segmented mirror telescopes.
\end{abstract}

Keywords: Telescopes, Segmented Mirrors, Optical Alignment, Phasing

\section{INTRODUCTION}

The Phasing Camera System (PCS) ${ }^{1}$ is a Shack-Hartmann (SH) based phasing camera responsible for the optical alignment of the Keck telescopes. The segments of the telescopes are routinely phased to $30 \mathrm{~nm}$ RMS surface piston error via the broadband (BB) phasing technique. ${ }^{2}$ While this level of accuracy is currently sufficient for the Keck telescope, it is not sufficient for TMT. TMT has a requirement to phase the segments to better than $10 \mathrm{~nm}$ RMS surface. We have demonstrated that the narrowband (NB) phasing technique ${ }^{3}$ can in principle achieve this accuracy. In fact this technique was originally developed for Keck, but subsequently abandoned in favor of the BB technique, which has a larger piston capture range and the advantage that it is not subject to aliasing effects.

As part of developing and demonstrating the required phasing accuracy for TMT, tests of NB phasing were conducted on the Keck telescopes. These tests revaled systematic errors that are wavelength dependent. ${ }^{4}$ These chromatic errors are on the order of $20 \mathrm{~nm}$ (surface) over the wavelength span from 600 to $900 \mathrm{~nm}$. Figure 1 shows the potential accuracy of NB phasing (left). The square root of the sum of the squares (RSS) values are $4.9 \mathrm{~nm}$, consistent with a measurement error of $10 \mathrm{~nm}$ for a single measurement. The right hand plot shows the chromatic effect which has an RSS value of $19 \mathrm{~nm}$. Extensive analysis has been performed ${ }^{4}$ and these chromatic effects do not arise from the algorithm, instrumentation, telescope, or segment coatings. The errors are related to the segment surfaces themselves. That is, a given segment Serial Number (SN) and edge is bad independent of which telescope and/or segment position it is installed in and the problem persists even if the segment is re-coated. This leaves only segment surface errors as the potential cause for the observed problems.

In Section 2 we investigate the radiometry from the Keck segment edges and quantify the amount of diffracted light. In Section 3 we present results from segment surface roughness measurements. Finally, in Section 4 we summarize the results, discuss the potential impacts of these errors on the future generation of giant segmented mirror telescopes (GSMTs) and discuss the next steps in this work.

Further author information: Send correspondence to Mitchell Troy, mtroy@jpl.nasa.gov. (C)2017. All rights reserved. 

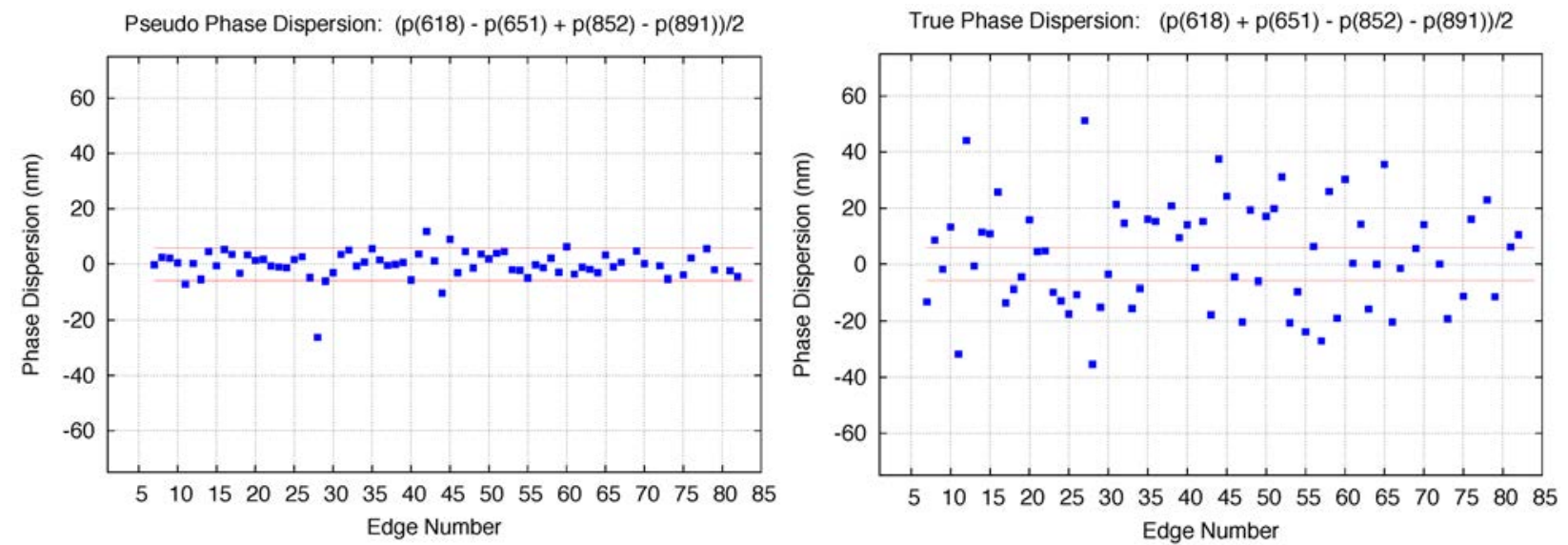

Figure 1. Two linearly independent linear combinations of phase measurements taken in four different filters. Left: The signs of the terms in the linear combination are chosen so that any wavelength dependence will approximately cancel out. Right: The signs are chosen so that any wavelength dependence will be enhanced. Horizontal lines represent the limits for random errors of the appropriate size. The larger scatter in the right plot is evidence of the systematic effect that we refer to as phase dispersion.

\section{ON-SKY MEASUREMENTS OF SEGMENT EDGES}

Measurements of the chromatic phase error are time consuming and by the associated data analysis is complicated. However, we have also observed a significant amount of diffracted light from some of the Keck segment edges. Figure 2 demonstrates that there is an anti-correlation between the phasing chromatic effect and the amount of light diffracted from a segment edge. This has the benefit that quantification and measurement of the diffracted intensity requires less observation time and minimal interpretation of the collected data.

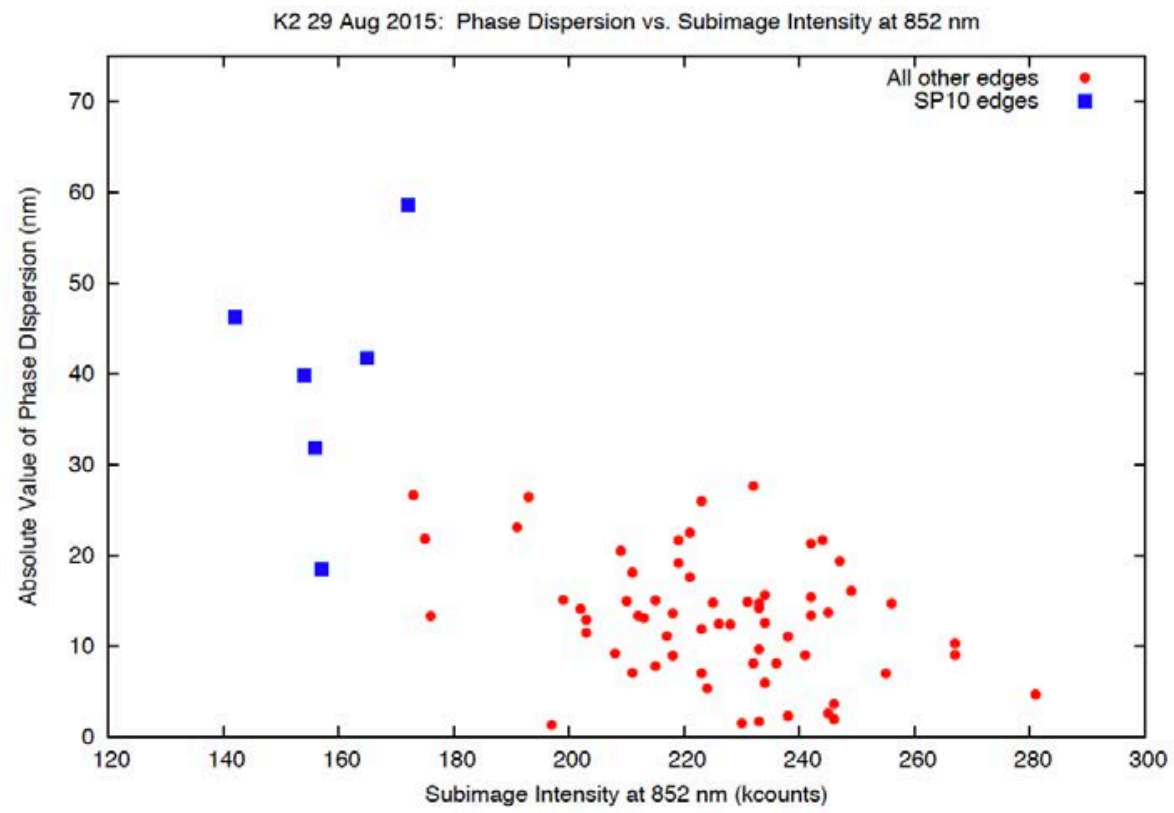

Figure 2. Phase dispersion is anti-correlated with phasing subimage intensity. The anti-correlation is significant even when the SP10 (which is SN9) edges are excluded.

The BB and NB phasing techniques work by measuring the interference between two adjacent subapertures 
on the segment edges. This is accomplished by using a mask at the pupil plane of PCS that defines $120 \mathrm{~mm}$ (at the primary mirror (M1)) diameter apertures that are half on each segment edge. The left hand side of Figure 3 shows the phasing mask and associated subapertures. There are 84 intersegment edges; the inner 6 are obscured by the tertiary mirror support and another 6 are under the secondary mirror (M2) supports and therefore ignored in this analysis. There are also 35 subapertures along the outer edge of the outer ring of segments. These subapertures are used to measure and register the re-imaged telescope pupil to phasing mask to a tolerance of better than $10 \mathrm{~mm}$ (at M1)

Using the telescope Active Control System (ACS) it is possible to tilt segments out of the segment stack and still control the remaining segments. Using this technique we can measure the intensity of the semi-circular subapertures that lie only on a single segment. On the right hand side of Figure 3 SP 8,10,12,14,16 and 18 are tilted out of the stack by $\sim 85$ arcseconds. The two white circles (on SPs 6 and 15) indicate phasing subapertures that look as expected: elliptical images with approximately half the intensity of the full phasing subapertures. The two red circles highlight two subapertures (on SP 20 and 36) that have low intensity and form images that indicate significant wavefront error over the semicircle.
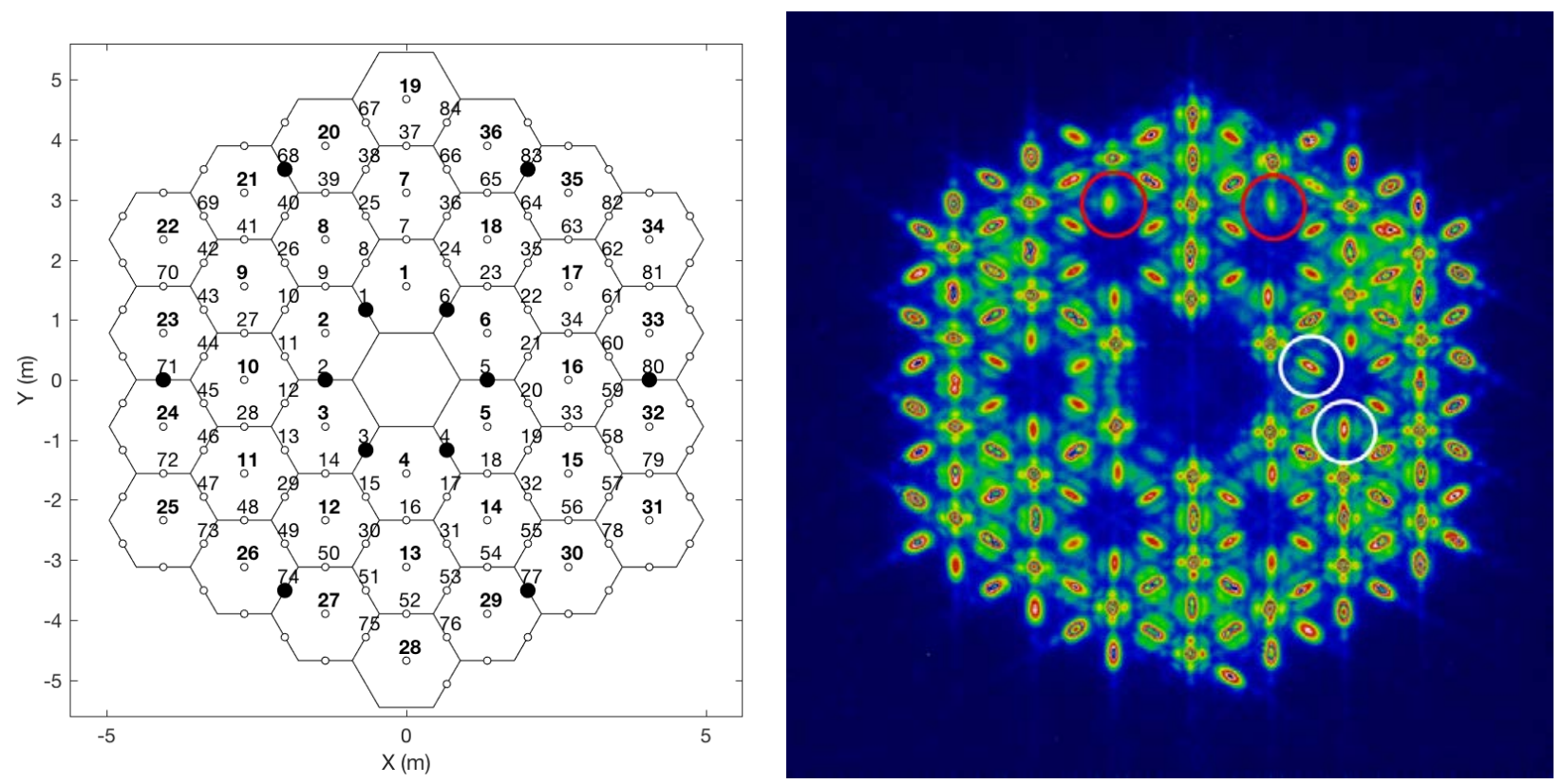

Figure 3. Left hand figure shows the pupil mask used in PCS for phasing of the Keck telescopes. The intersegment subapertures are $120 \mathrm{~mm}$ in diameter (at M1). The right hand figure is a phasing image taken with SPs 8,10,12,14,16 and 18 tilted out of the stack.

Figure 4 shows the images formed by starlight from a single segment passing through the phasing camera optics. The image on the left is a good segment and on the right one of the worst segments (SN9, currently SP14 in Keck II). These images provide a clear demonstration that some of the segment edges are diffracting light to large angles. On two of the edges the wavefront error is so large that no well-defined Point Spread Function (PSF) is formed.

\subsection{Data Collection and Analysis}

We have collected data on phasing subaperture intensities on both Keck telescopes using the above technique. We have data from Keck I on UT 02/11/2017 and data from two nights on Keck II (UT 02/08/2017 and 6/11/2017). The data were collected using a filter centered on $852 \mathrm{~nm}$ with a Full Width at Half Maximum (FWHM) of $\sim 35 \mathrm{~nm}$ on stars of visual magnitudes from 2.3 to 4.5 and 15 seconds integration time per frame. In general, 3 frames of data were taken in each configuration. On Keck II data were taken with the phasing subapertures 

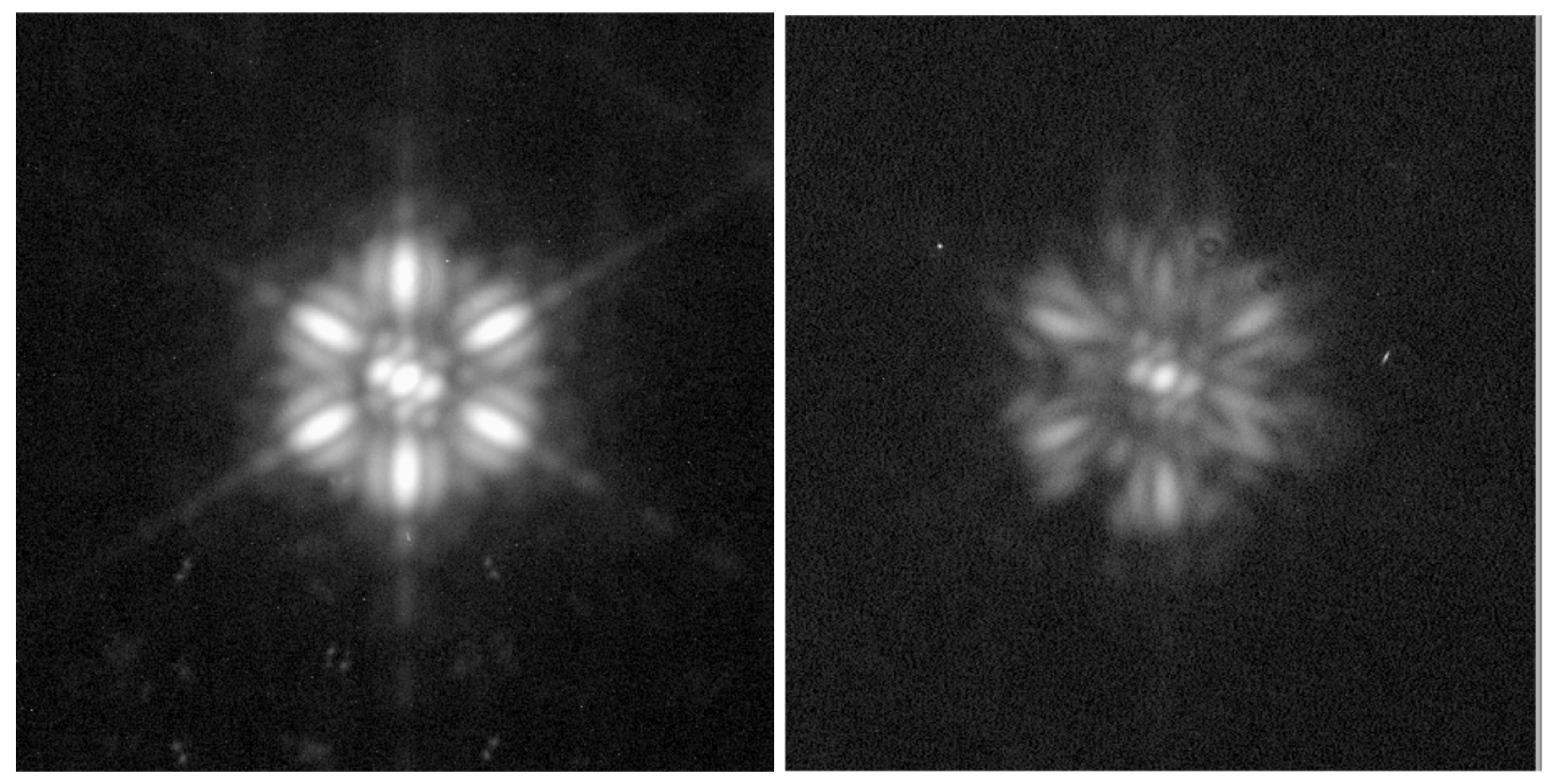

Figure 4. Images above are diffraction patterns formed by light from single segments passing through the phasing camera optics with the phasing mask. On the left a good segment and on the right one of the worst segments (SP14/SN9). Evidently the edges on the latter are diffracting light well beyond the diffraction cores.

in their nominal location (centered left/right on a segment edge) and also shifted left/right by $\pm 200 \mathrm{~mm}$ on a segment edge.

Each subimage was extracted and the circular aperture photometry calculated over a diameter of 48 pixels, which is equivalent to \pm 3.55 arcseconds on the sky. A surrounding aperture of width 2 pixels was used for background subtraction. Multiple methods were investigated for normalization of the data; all produced similar results and the same conclusions. The results presented in this paper are normalized by the median intensity of the interior segment subimages in order to correct for non-photometric conditions. Finally the results were multiplied by two in order to account for the difference in collecting area of the half-illuminated subapertures.

\subsection{Results}

Figure 5 shows the normalized edge intensity for the 72 segments we have measured to date. For each segment the plot shows the mean and standard deviation for all of the edge measurements on that segment. About $25 \%$ of segments have edges with a significant reduction $(>20 \%)$ in intensity within \pm 3.5 arcseconds. Figure 6 shows the same normalized intensity as Figure 5 but only for the horizontal segment edges, with measurements of the phasing subaperture on the segment center and shift left and right along the segment edge. Over $60 \%$ of measured segments show a significant variation along a segment edge. We have also made measurements with the phasing spots on the interior of the segments and no subimage intensity variations were observed. Thus, these effects appear to be limited to the outer $\sim 100 \mathrm{~mm}$ of the segment edges.

For each segment measured we have calculated the mean intensity of all measured edges as well as the minimum value. Figure 7 shows the cumulative probability that an edge has less than a given intensity. On average the mean edge intensity across all measured segments is 0.99. consistent with expectations. However, $7 \%$ (or 5 ) of the measured segments have average edge values less than $80 \%$ of the expected value and $25 \%$ (or 18) have at least one edge less than this value.

\subsection{Correlation of Subimage Intensity with IBF Data}

IBF was performed on the Keck segments starting in July, 1990, just months after the IBF machine was received at what was then Kodak. ${ }^{5}$ Surface measurements of the segments before IBF were made at a different location 


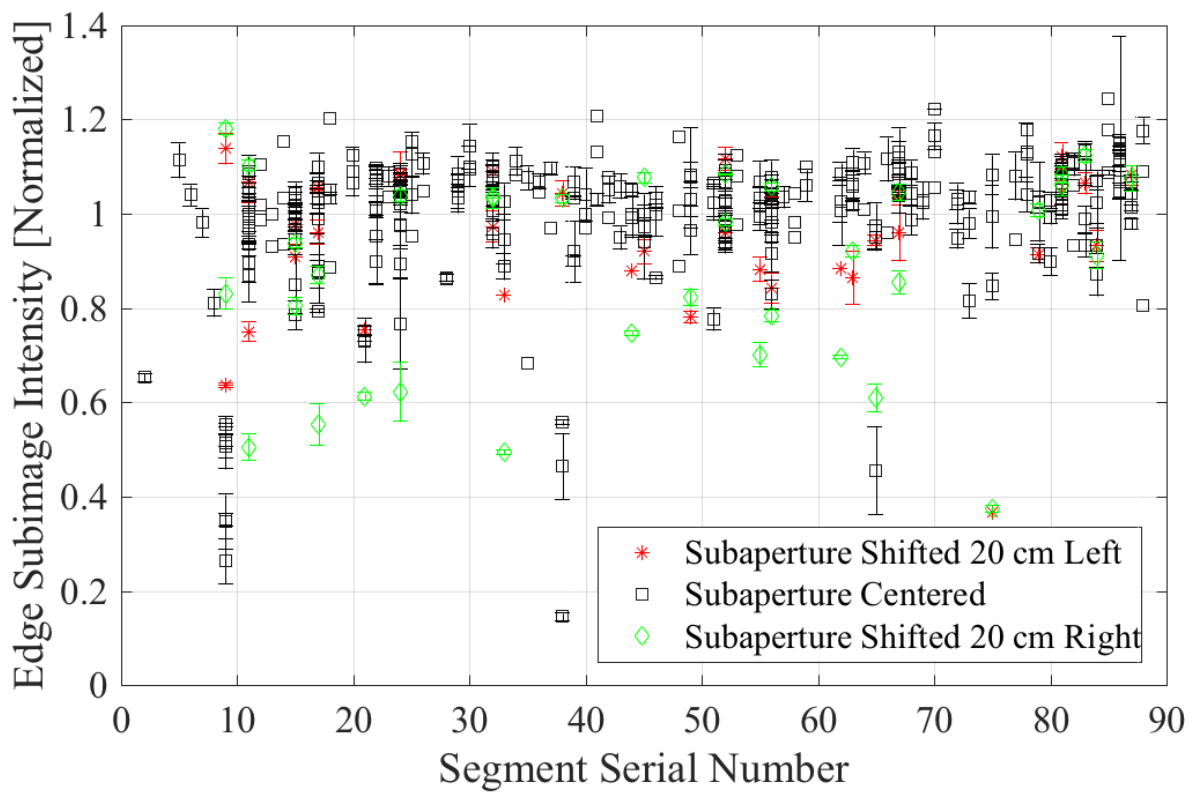

Figure 5. Normalized subimage intensity for each edge subaperture location measured as a function of segment SN. The error bars indicate the RMS of the values for a given subaperture location.

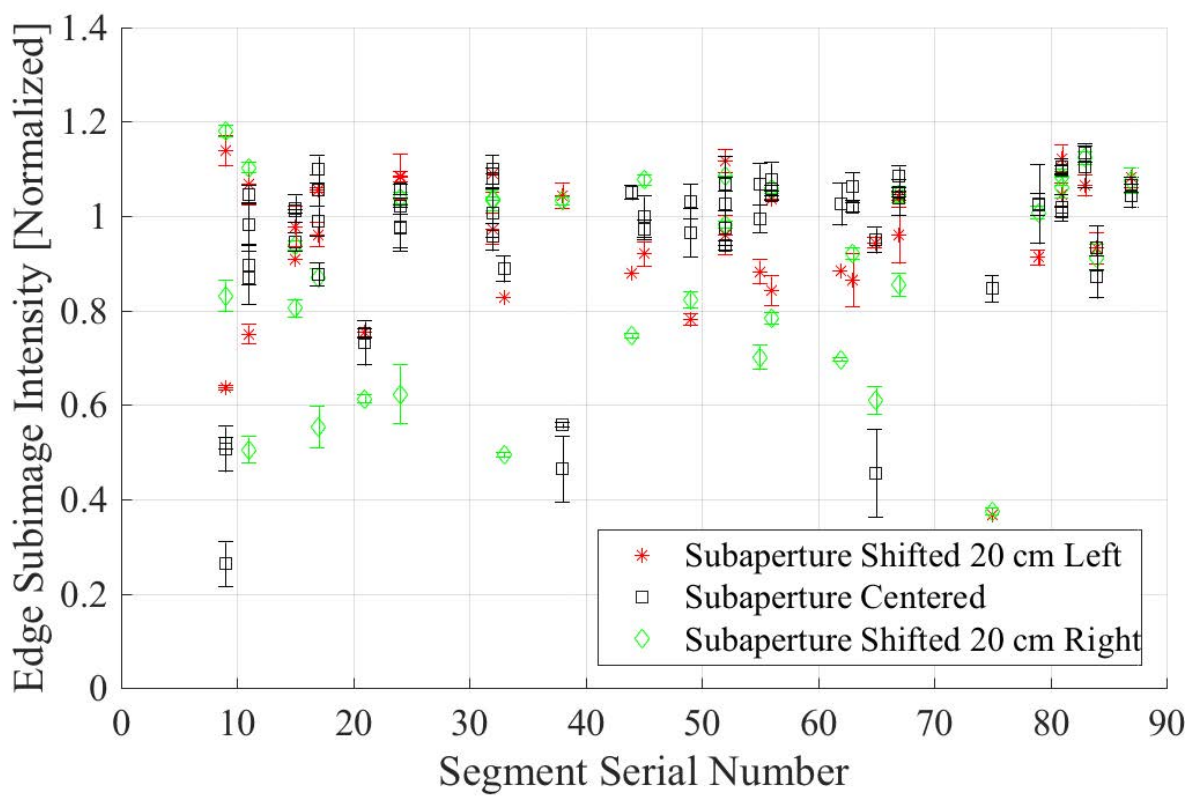

Figure 6. Normalized subimage intensity for each edge subaperture on a segment horizontal edge. The error bars indicate the RMS of the values for a given subaperture location. There is a significant variation in intensity over the three locations measured along the segment edges.

and by a different company (Itek). The original summary IBF data for each segment is still avaiable and includes for each segment: ${ }^{6}$

- the month and year IBF was performed 


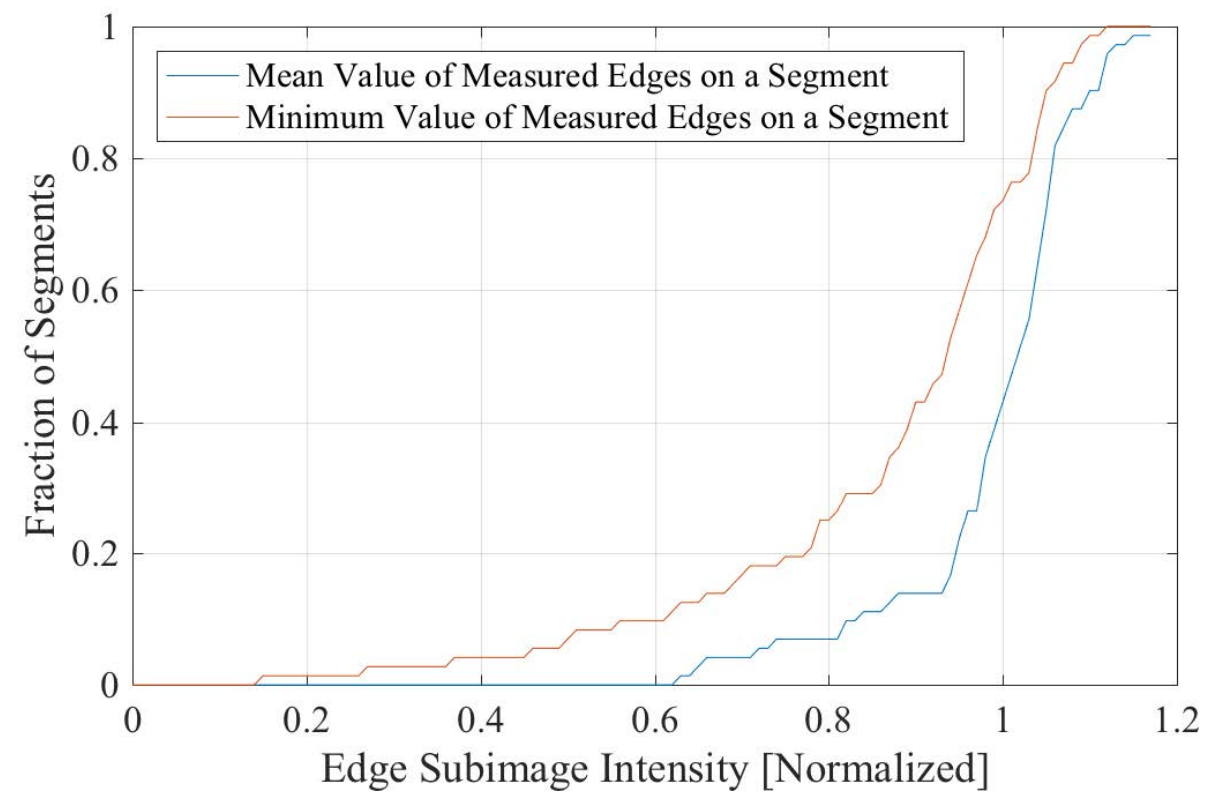

Figure 7. Cumulative probability that the mean (or minimum) edge on a segment is less then a given intensity.

- RMS surface error and $80 \%$ enclosed energy before IBF with and without warping harness correction

- RMS surface error and $80 \%$ enclosed energy after IBF with and without warping harness correction for those segments that were measured (not all were measured)

- the number of hours IBF was performed

- the amount of material removed by IBF

Figure 8 plots the subimage intensity vs. year the IBF was performed. IBF was not performed on the two segments plotted at time zero in the Figure. Segments with significant subimage intensity problems were generated throughout the time IBF was performed and are not just associated with the first few segments. We investigated correlations of subimage intensity with the other IBF data and no correlations were found.

\section{SURFACE MEASUREMENTS WITH 4D NANOCAM}

When first observed it was thought that the low subimage intensities might be coming from problems in the segment coatings. However, careful measurements with a reflectometer as well as analysis of witness samples showed this not to be the case. The mirror coatings all behaved as expected. The reflectometer has an acceptance angle of \pm 3 degrees, but the PCS subimages only capture light from \pm 3.5 arcseconds. Thus, the scatter must be caused be aberrations near the segment edge with spatial periods on the order of a few $\mathrm{cm}$ or lower. As a result, surface roughness measurements of the segments were conducted to determine if this was the cause of the observed problems.

\subsection{Data Collection}

In September, 2016 surface roughness measurements were made of 3 segments (SN 9, 27, and 50). SN 9 was measured twice: with an approximately two year old coating and without any coating. SN 27 was measured without a coating and SN 50 was measured with an approximately two year old coating. Figure 9 shows the 4D Technology NanoCam making a measurement on a segment. The measurements were made in a lab on the Keck summit with the segments horizontal and mounted on their handling carts. Measurements were made at $\sim 7$ locations along the edges of each segment as well as at four locations interior to the segment (at the center 


\section{Minimum Segment Edge Intensity vs Year Segment was Ion Figured [Time of Zero Indicates Segment Not IBFed]}

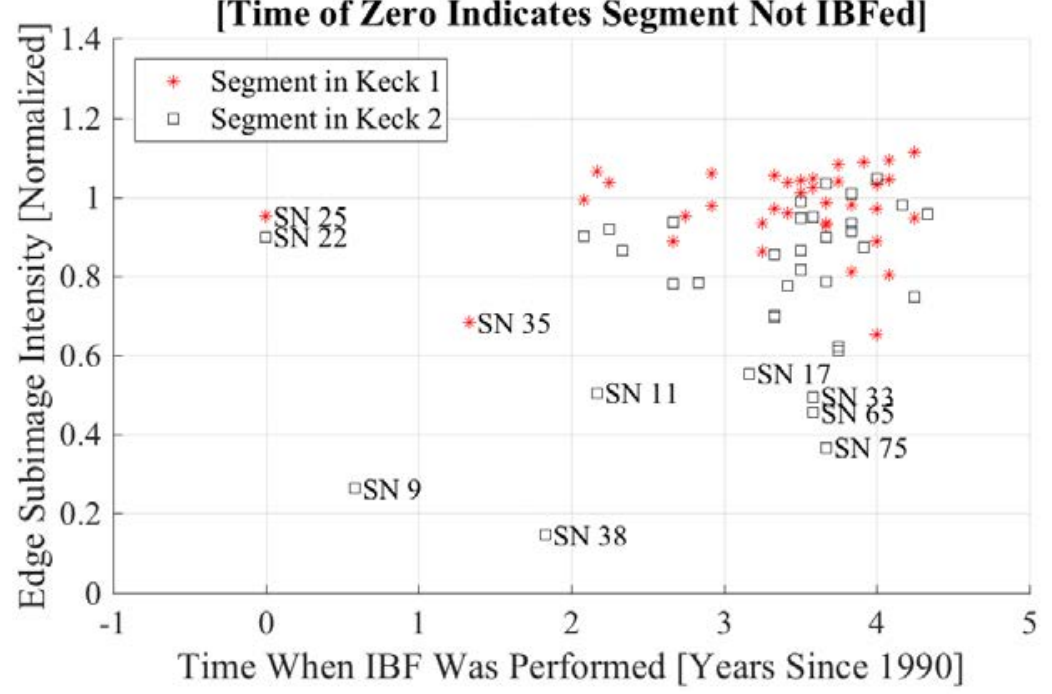

Figure 8. Normalized subimage intensity of the minimum value for a given edge on the indicated segment as a function of year IBF was performed. The two segments plotted at time zero did not undergo IBF.

of the segment edge). In total over 200 surface measurements were made. Table 1 summarizes the relevant parameters for the objective magnifications used during the measurements. The largest field of view that we could measure with the NanoCam was $4.4 \mathrm{~mm}$, which means we were insensitive to errors with spatial periods larger than $\sim 2 \mathrm{~mm}$.

\begin{tabular}{|c|c|c|}
\hline Magnification & $2 \mathrm{x}$ & $20 \mathrm{x}$ \\
\hline Field of View (mm) & 4.4 & 0.44 \\
\hline Spatial Sampling (um) & 3.7 & 0.37 \\
\hline
\end{tabular}

Table 1. Specifications of NanoCam for objective magnifications used during testing.

\subsection{Results}

Figure 10 shows example surface roughness measurements at a magnification of 20x. Figure 11 shows the median surface error over each segment edge (A-F). These figures show that the uncoated segments have surface roughness values of less than $\sim 3 \mathrm{~nm}$. This is even after $25+$ years in the telescope and multiple re-coatings. The segments with coatings have a higher measured surface roughness, likely due to the $\sim 2$ year old coatings. SN 9 does seem to have larger errors, which may be related to the significant amount of time needed for IBF, ${ }^{7}$ a factor of 11 and 3.5 more than for SN 50 and SN 27, respectively. The observed surface roughness cannot explain the chromatic or subimage intensity effects.

\subsection{IBF Plateaus}

During IBF the segments were supported underneath by the use of three "J-Hooks", as shown in the left hand side of Figure 12. These J-Hooks were centered on the segment edge and were approximately $50 \mathrm{~mm}$ wide and $25 \mathrm{~mm}$ across. As a result the area under these supports was not exposed to the IBF beam and in at least some cases plateaus were left behind. The right hand side of Figure 12 shows a measurement of one of these plateaus on SN 9. The plateaus were observed on at least 4 of the edges of SN 9, but on none of the other two segments. 


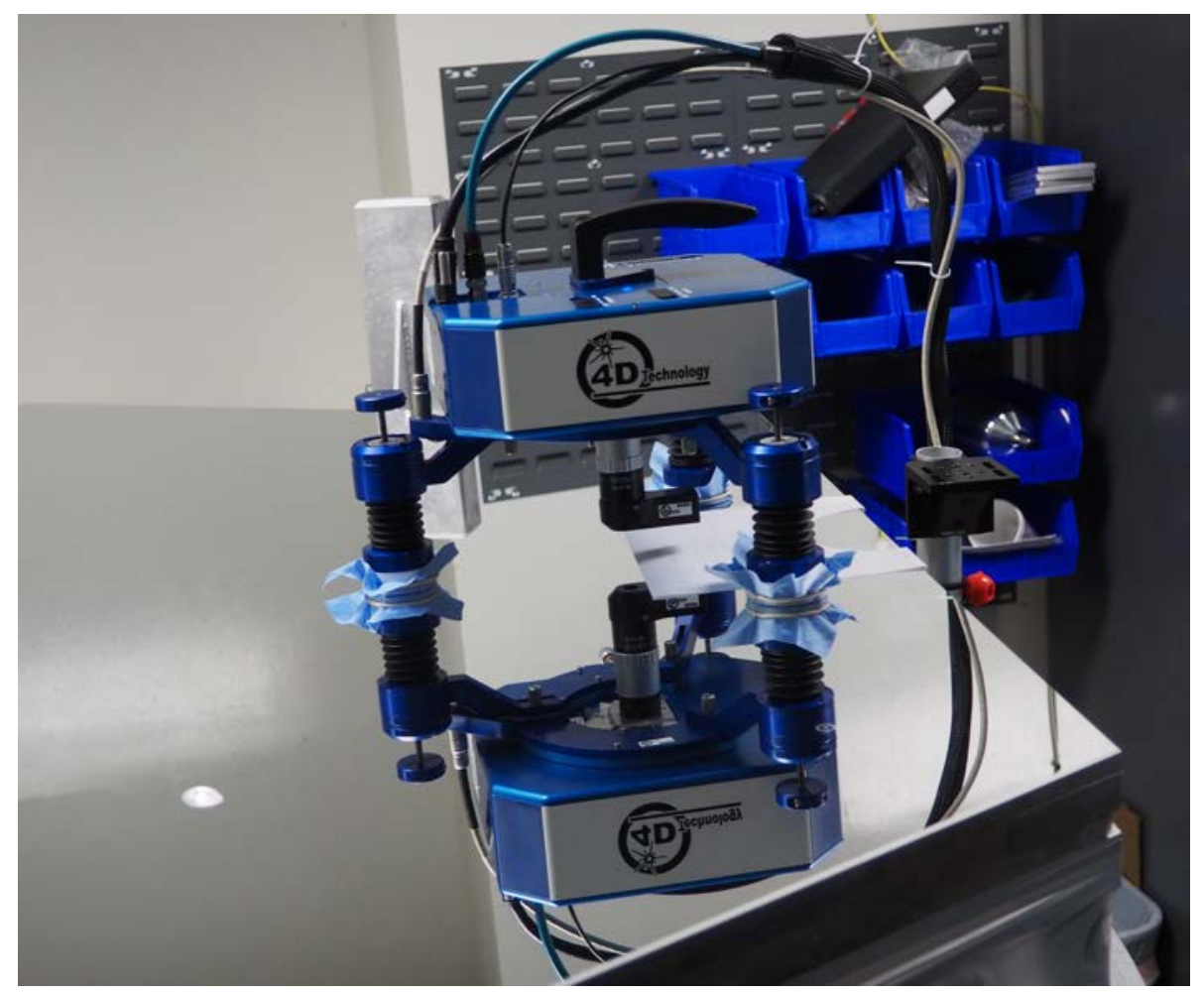

Figure 9. Photograph of the NanoCam making a surface roughness measurement on a Keck segment.
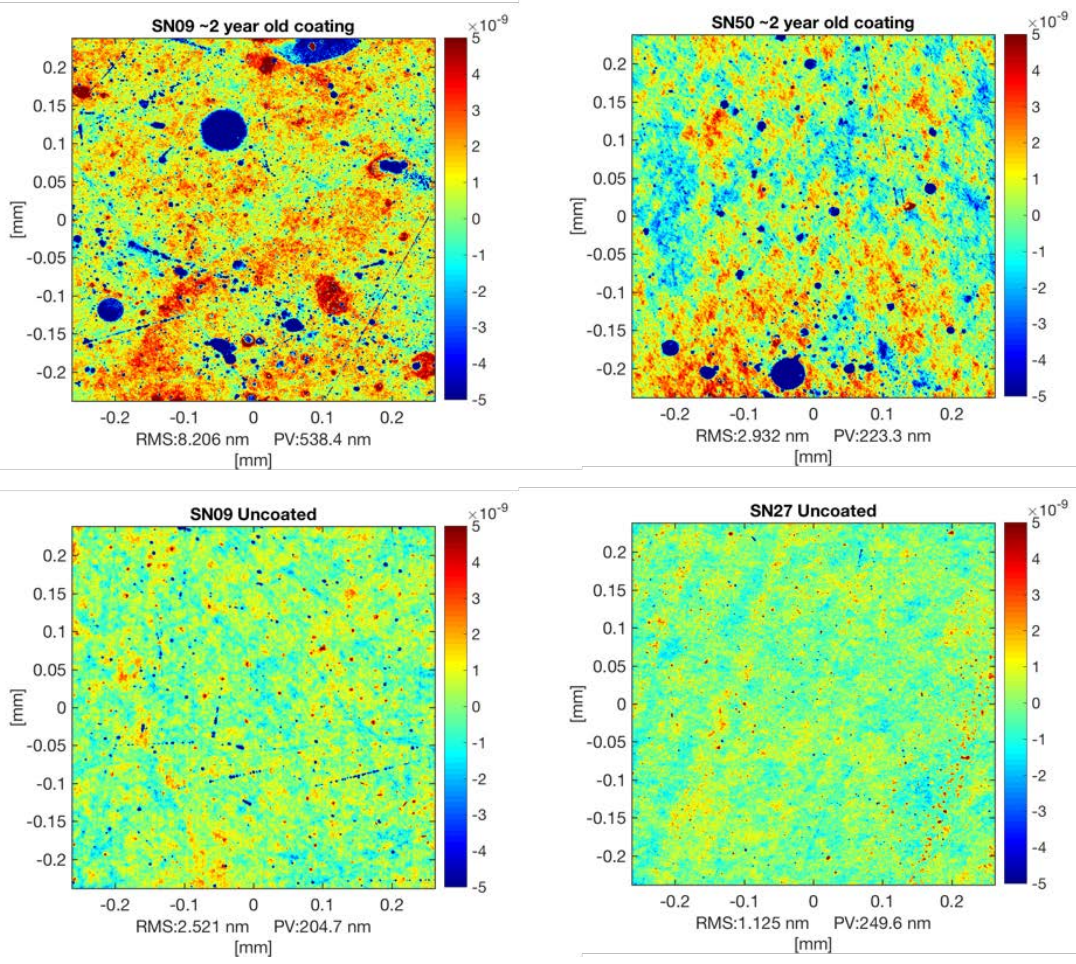

Figure 10. Example measurements of surface roughness at a magnification of 20x. See text for description. 


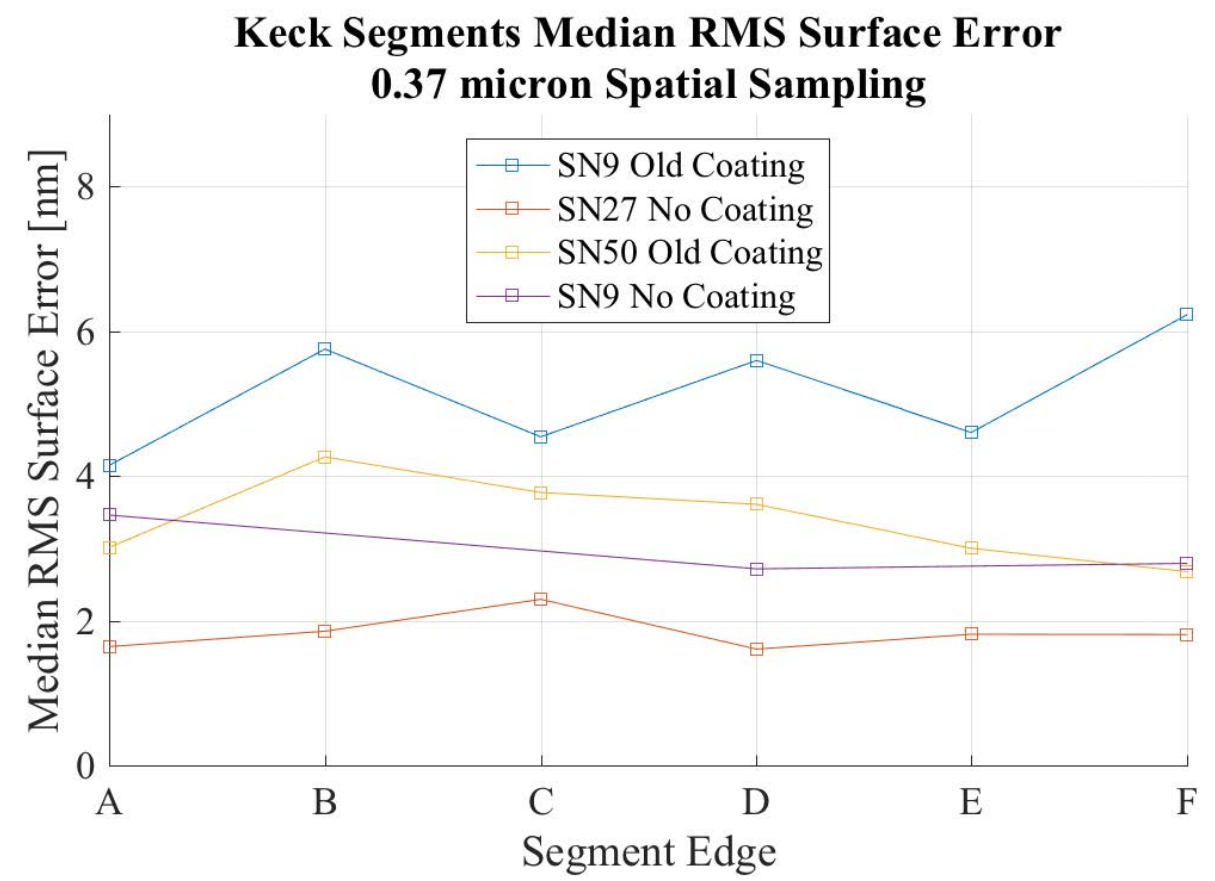

Figure 11. Median surface roughness for each segment edge measured.

The plateau is a relatively flat suface with that is $\sim 500-800 \mathrm{~nm}$ tall and the edge rises over a distance of $\sim 0.7 \mathrm{~mm}$. In December, 1991 the configuration of the J-Hooks was apparently changed to be two 1 inch square brackets located at $\pm 50 \mathrm{~cm}$ from the center of the segment edge in order to insure that the supports were outside of the area of the segment used for phasing measurements. ${ }^{8}$ This would have occurred just after SN 38 was processed. Simulations have shown that these J-Hooks could not cause either the low subimage intensity or the chromatic phase dispersion. In addition Figure 6 demonstrates that there is significant variation of subimage intensity along a segment edge and this also argues against any significant effect from these supports. Nevertheless, supporting the segment from the optical surface during IBF is less than ideal and will not be done for the TMT segments.

\section{CONCLUSIONS AND FUTURE WORK}

In summary the Keck segment edges diffract a significant fraction of light out beyond \pm 3.5 arcseconds. This effect occurs only near the segment edges. We have also previously shown that subapertures with low subimage intensity correlate to those with large chromatic phase dispersion. ${ }^{4}$ Although the TMT segments should not be supported from the optical surface during IBF, the plateaus seen in some of the Keck segments from the J-Hook supports are not the cause of these effects.

The Keck segments when produced 25 years ago were both the first ever made via Stress Mirror Polishing (SMP) and the first optics to ever be ion beam figured. The segments exceeded all requirements specified at the time they were polished. The surfaces have maintained very good quality over these 25 years despite the many re-coatings of the mirror. However, as we move forward the requirements on telescope optics have increased. If the TMT segments (or those of any GSMTs) were like the Keck segments then:

- The required integration time for background limited observing would increase by $10 \%$.

- Segment piston errors would dominate the telescope high-contrast error budget.

- The Adaptive Optics (AO) corrected Strehl at H-band would decrease by 5\%.

- 1.3 additional nights per year would be needed for engineering time due to the required use of a slower and less accurate phasing method. 


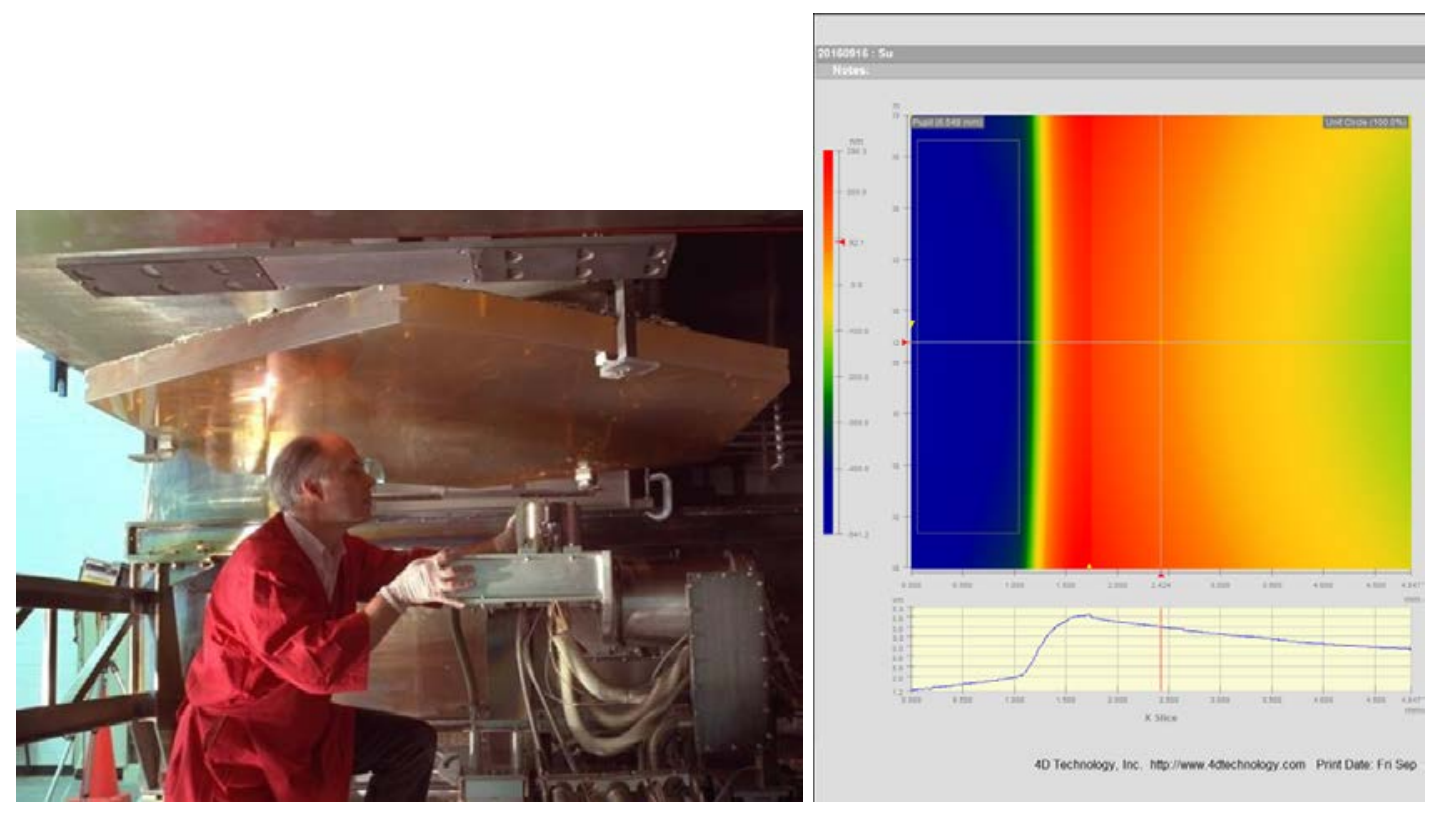

Figure 12. On the left an image of a Keck segment in the IBF chamber. The first three segments were supported at the center of three of the segment edges. After that it appears that the segments were supported by two pads at $\pm 50 \mathrm{~cm}$ from the center of three of the edges. On the right is the measured phase of the edge of a plateau on SN 9 . See the text for a detailed description.

These effects are likely caused by IBF residuals with a spatial period of 1-3 $\mathrm{cm}$ and 10-20 nm RMS amplitude (surface). Measurements of the Keck segment edges with an interferometer are currently being planned to confirm this theory. We are also in discussions with Harris and TMT to better understand the root cause of these effects and to insure that they do not occur on the TMT segments.

\section{ACKNOWLEDGMENTS}

The research was carried out in part at the Jet Propulsion Laboratory, California Institute of Technology, under a contract with the National Aeronautics and Space Administration.

The authors gratefully acknowledge the support of the TMT collaborating institutions. They are the California Institute of Technology, the University of California, the National Astronomical Observatory of Japan, the National Astronomical Observatories of China and their consortium partners, the Department of Science and Technology of India and their supported institutes, and the National Research Council of Canada. This work was supported as well by the Gordon and Betty Moore Foundation, the Canada Foundation for Innovation, the Ontario Ministry of Research and Innovation, the Natural Sciences and Engineering Research Council of Canada, the British Columbia Knowledge Development Fund, the Association of Canadian Universities for Research in Astronomy (ACURA), the Association of Universities for Research in Astronomy (AURA), the U.S. National Science Foundation, the National Institutes of Natural Sciences of Japan, and the Department of Atomic Energy of India.

We would like to thank the W. M. Keck Observatory for their support of the surface roughness measurements and for providing the telescope engineering time needed for the on-sky measurements.

\section{REFERENCES}

1. Chanan, G., Nelson, J., Mast, T., Wizinowich, P. L., and Schaefer, B. A., "W.M. Keck Telescope phasing camera system," Proc. SPIE 2198, 1139-1150 (June 1994). 
2. Chanan, G., Troy, M., Dekens, F. G., Michaels, S., Nelson, J., Mast, T., and Kirkman, D., "Phasing the mirror segments of the Keck telescopes: the broadband phasing algorithm," Applied Optics 37, 140-155 (Jan. 1998).

3. Chanan, G., Ohara, C., and Troy, M., "Phasing the mirror segments of the Keck telescopes: the narrowband phasing algorithm," Applied Optics 39, 4706-4714 (Sep. 2000).

4. Chanan, G., Troy, M., and Raouf, N., "Phasing the segments of the Keck and Thirty Meter Telescopes via the narrowband phasing algorithm: chromatic effects," Proc. SPIE 9906, 1184-1191 (Jul. 2016).

5. Allen, L. N., Keim, R. E., Lewis, T. S., and Ullom, J. R., "Surface error correction of a Keck 10-m telescope primary mirror segment by ion figuring," Proc. SPIE 1531, 195-204 (Jan. 1992).

6. Personal Communication with Lynn Allen at Harris Corporation (2016).

7. Allen, L. N., "Progress in ion figuring large optics," Proc. SPIE 2428, 237-247 (Jul. 1995).

8. Personal Communication with Peter Wizinowich at Keck Observatory (2017). 